\title{
PLANEJ AMENTO DA ASSISTÊNCIA DE ENFERMAGEM AO PACIENTE COM CÂNCER: REFLEXÃO TEÓRICA SOBRE AS DIMENSÕES SOCIAIS
}

\author{
Planning of the assistance of nursing to the patient with cancer: theoretical reflection \\ on the social dimensions \\ Planificación de cuidados de enfermería del paciente con cáncer: reflexión teórica sobre \\ las dimensiones sociales
}

Rita de Cássia Velozo da Silva

Enêde Andrade da $\mathrm{Cruz}^{2}$

\begin{abstract}
RESUMO
Artigo de reflexão que enfoca as dimensões sociais envolvidas no planejamento da assistência de enfermagem ao paciente com câncer, utilizando como base conceitos da Teoria das Representações Sociais. 0 câncer traz uma série de implicações que interferem na saúde do indivíduo, gerando repercussões não só orgânicas, mas também afetivas, emocionais e sociais. Nessa perspectiva, as autoras buscam fornecer uma contribuição à categoria, no sentido de subsidiar discussões sobre o tema, visto que, ao planejar a assistência, a enfermeira determina prioridades, define intervenções, para organizar e orientar as ações assistenciais de sua equipe com vistas aos resultados pretendidos. Essa prática, embasada na assistência humanizada e no respeito ao outro, proporciona o fortalecimento do vínculo enfermeira-paciente-família. Os resultados apontam para a necessidade de ampliação dessas reflexões e para o reconhecimento das condições e processos de trabalho que exprimam e articulam a relação entre a objetividade da prática e a subjetividade dos profissionais envolvidos.
\end{abstract}

Palavras-chave: Enfermagem Oncológica. Planejamento de Assistência ao Paciente

\begin{abstract}
Article discussion that focuses on the social dimensions involved in the planning of nursing care to patients with cancer, using as basic concepts of the Theory of Social Representations. Cancer brings a number of implications that affect the health of the individual, creating an impact not only organic, and affective, emotional and social. From this perspective, the authors seek to provide a contribution to the profession, as an aid to discussions on the topic, as when planning assistance, the nurse determines priorities, sets out measures to organize and guide the actions of their care team with a view to the results required. This practice, grounded in humanizing and respect for others, provides a stronger link nurse-patient-family. The results indicate the need to expand these ideas and to recognize the conditions and work processes to express and articulate the relationship between objectivity and subjectivity of the practice of professionals.
\end{abstract}

Keywords: Oncology nursing. Patient care planning.

\section{Resumen}

Artículo de reflexión que enfoca las dimensiones sociales involucradas en la planificación de los cuidados de enfermería a pacientes con cáncer, basándose en conceptos de la Teoría de las Representaciones Sociales. El cáncer trae una serie de consecuencias que afectan la salud del individuo, creando un impacto no sólo orgánico, pero afectivo, emocional y social. Desde esta perspectiva, las autoras tratan de aportar una contribución a la profesión, en el sentido de subsidiar debates sobre el tema, una vez que, con la ayuda en la planificación, la enfermera determina prioridades, establece medidas para organizar y orientar las acciones de atención de su equipo con miras a los resultados requeridos. Esta práctica, basada en la humanización y en el respeto por los demás, proporciona un fortalecimiento del vínculo enfermera-paciente-familia. Los resultados indican la necesidad de ampliar estas ideas y para el reconocimiento de las condiciones y procesos de trabajo para expresar y articular la relación entre la objetividad y la subjetividad de la práctica de los profesionales.

Palabras clave: Enfermería oncológica. Planificación de atención al paciente.

'Enfermeira, mestra, docente da Faculdade Maria Milza e da Faculdade São Camilo, supervisora da Central Estadual de Regulação. Salvador - BA. Brasil. Email: velozoped@yahoo.com.br,2Doutora, docente do Programa de Pós-Graduação da Escola de Enfermagem da Universidade Federal da Bahia, membro do GEPASE e coordenadora do Núcleo de Pesquisa e Extensão em Enfermagem em Centro Cirúrgico e Central de Material NUPESCC. Salvador-BA. Brasil. Email: enedeeac@ig.com.br 


\section{INTRODUÇÃO}

0 câncer representa um conjunto de mais de cem doenças, sendo, atualmente, a segunda causa de mortalidade, mesmo nos países desenvolvidos, culminando em altos investimentos em custos hospitalares, medicamentos e tecnologias. A incidência da doença cresce também no Brasil, em um ritmo que acompanha o envelhecimento populacional decorrente do aumento da expectativa de vida. É um resultado direto das transformações globais das últimas décadas, que alteraram a situação dos povos pela urbanização acelerada, novos modos de vida, novos padrões de consumo. ${ }^{1}$

Entretanto, o aumento da incidência de casos de câncer não decorre exclusivamente do crescimento real da doença, mas se deve também às novas tecnologias desenvolvidas para maior acurácia diagnóstica e ao aperfeiçoamento dos meios de tratamento. 0 que se observa é que 0 aumento na duração das doenças, com a expansão daquelas crônicas como o câncer, impõe novos modos de condução do tratamento, novas modalidades de assistência e novos percursos para os pacientes em busca de sua reabilitação.

Apesar das necessidades evidenciadas, o fluxo de ações ainda é lento para o crescimento da demanda, principalmente no que diz respeito à expansão de leitos hospitalares e estruturação de atendimento domiciliar em cuidados paliativos. Nesse sentido, aliado ao diagnóstico tardio, existe a demora no início do tratamento, o que pode limitar as chances de sobrevivência desses pacientes.

Perceber o paciente com câncer traz significados diversos, mudanças de valores, crenças e atitudes que demandam intervenções apropriadas e individualizadas, para minimizar a ameaça à sua integridade física e psíquica, o que leva a enfermeira, e demais profissionais de sua equipe, a confrontar-se com sua própria vulnerabilidade e finitude.

Assistir ao paciente com câncer vai além de uma prescrição de cuidados: envolve acompanhar sua trajetória e de sua família, desde os procedimentos diagnósticos, tratamento, remissão, reabilitação, possibilidade de recidiva e fase final da doença, ou seja, vivenciando situações do momento do diagnóstico à terminalidade.

0 planejamento, pois, caracteriza-se como a primeira etapa de qualquer atividade assistencial da enfermagem. Implica estabelecer os objetivos da assistência, analisar as consequências que poderiam advir de diferentes atuações, optar entre alternativas, determinar metas específicas a serem atingidas e desenvolver estratégias adequadas à execução da

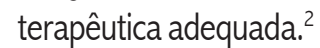

Essa prática sofre interferência dos sentimentos, atitudes, valores e crenças que a enfermeira e os profissionais de enfermagem trazem para o seu fazer cotidiano, fundamentados no conhecimento científico, mas que muitas vezes são transformados no desempenho profissional. As interações referentes às tarefas, às relações entre os indivíduos do grupo que ocorrem no cotidiano podem conduzir à criação de ideias compartilhadas ou senso comum, caracterizadas como representações sociais (RS).

0 senso comum caracteriza-se como uma modalidade de conhecimento particular que tem por função a elaboração de comportamentos e a comunicação entre os indivíduos. Nesse sentido, a RS diz respeito ao que os seres humanos pensam considerando que são sujeitos que pensam de forma autônoma, produzem e comunicam suas ideias no seu meio social, o que irá influenciar, de forma efetiva, a maneira como se desenvolvem suas relações sociais. ${ }^{3}$

Assim, refletir sobre a prática da enfermeira no planejamento da assistência pode evidenciar elementos que são próprios do contexto e também aqueles que resultam do processo de comunicação que é próprio do grupo no desenvolvimento de suas atividades junto ao paciente com câncer.

Consideramos que, ao assumir uma equipe de trabalho, a enfermeira tem uma responsabilidade relevante, visto que o planejamento da assistência envolve a tomada de decisões e ações voltadas para a resolução dos problemas identificados, cujas ações são executadas, em sua maioria, pelos demais membros da equipe de enfermagem. É imprescindível, portanto, que este planejamento seja coerente com as condições e valores sociais da pessoa enferma, sua família e grupo social, pois é nesse contexto que a situação da doença será vivenciada.

Diante do exposto, delimita-se como objetivo deste estudo refletir sobre as dimensões sociais envolvidas no planejamento da assistência de enfermagem ao paciente com câncer, a partir da aproximação da autora na gestão de uma equipe de enfermagem e na assistência ao paciente com câncer, onde foram observados aspectos complexos e singulares.

Daí a opção por um estudo no sentido de aprofundar o conhecimento e possibilitar reflexões sobre a subjetividade vivenciada no planejamento da assistência pela enfermeira, atentando para as dimensões sociais envolvidas nesse processo, com base nos aportes teóricos da Teoria das Representações Sociais, ${ }^{3}$ por considerá-la como importante instrumental teórico para discutir sobre estes aspectos subjetivos.

Essa Teoria tem sido utilizada para estudar como os indivíduos e os grupos sociais se apropriam dos objetos do cotidiano, oferecendo-Ihes sentidos e lugares sociais que dizem tanto da história quanto da dinâmica dos relacionamentos intragrupais e intergrupais, além de mostrar que o processo saúde-doença também é atravessado por elementos culturais, sociais e econômicos, sendo compreendido diferentemente pelos vários atores que dele participam..$^{4-5}$ 


\section{O PACIENTE COM CÂNCER E SUA FAMÍLIA}

0 impacto que uma doença provoca sofre interferência de alguns fatores, como sua Adoecer por câncer é uma experiência que ultrapassa o corpo físico, embora nele se instale e desenvolva sentimentos e significados, estes interpretados e reinterpretados tanto pela pessoa doente quanto por aquelas com as quais convive. ${ }^{6}$

A compreensão desses aspectos pode direcionar 0 profissional de enfermagem a olhar para além da pessoa doente. Isto porque cronicidade, a repercussão que esta pode gerar, quer seja do ponto de vista das alterações orgânicas ou em relação às questões afetivas, emocionais e sociais. $\mathrm{Ou}$ seja, a doença traz em si um significado individual, que é pessoal, mas também tem um significado coletivo, que é social.

0 câncer representa mais que uma dor física e um desconforto. Ele interfere nos objetivos de vida do paciente, em sua família, seu trabalho e renda; sua mobilidade, sua imagem corporal, e seu estilo de vida podem ser drasticamente alterados. Essas mudanças podem ser temporárias ou permanentes e produzem repercussões que afetam a todos, inclusive aos profissionais responsáveis pela assistência, pois cada pessoa tem formas únicas de ver o mundo, e, consequentemente, de lidar com a doença.

A doença oncológica mobiliza muitos sentimentos na família, que muitas vezes quer esconder do paciente o seu diagnóstico, o que pode comprometer o processo de comunicação com o paciente e com a equipe e limitar a autonomia do paciente. A Enfermagem precisa, contudo, considerar os preceitos legais exigidos à sua categoria, onde devem ser respeitados os valores éticos e seus princípios fundamentais, pois, embora os avanços tecnológicos propiciem um poder de intervenção sobre a vida das pessoas, requer observar as repercussões destas para os indivíduos e para a sociedade.

Sabe-se que o estigma desse tipo de doença grave está presente nos comportamentos das pessoas e sujeita a preconceitos, que, a depender do contexto onde a relação paciente-profissional acontece, existem aspectos que influenciam nas reações à doença e ao próprio tratamento. Tais reações parecem estar relacionadas também às atitudes que os profissionais adotam diante da doença.

Estudos ${ }^{4,7,8}$ apontam para uma representação social compartilhada por diferentes grupos sociais em que o câncer equivale a uma sentença de morte. Os avanços tecnológicos no seu diagnóstico e tratamento parecem não ter sido suficientes no sentido de transformar o significado e a imagem que socialmente impregnam a ideia do câncer.

Diante disso, ao colaborar no tratamento desse paciente, a enfermeira e sua equipe têm diante de si diversas formas de se aproximar e interferir na vida dele, visto que suas atividades são diversificadas e vão desde a quimioterapia curativa até os cuidados paliativos, mostrando a amplitude de possibilidades de intervenção, ao tempo em que demonstra a necessidade de conhecimento, competência, responsabilidade, acolhimento e compromisso no desenvolvimento de suas ações.

$E$, muito embora a hospitalização seja muitas vezes imprescindível ao tratamento, à prestação de cuidados mais complexos e à recuperação da pessoa doente, o hospital pode tornar-se um ambiente hostil, frio, limitador da presença mais assídua de pessoas próximas, ${ }^{9}$ em vista do tempo de permanência dos pacientes com câncer, que geralmente é prolongado.

Sob esta perspectiva, ao considerar o paciente em todas as suas peculiaridades, a enfermagem participa do processo de adoecer e morrer dessas pessoas, cuidadas muitas vezes por seus familiares, em que ambos (paciente e família) podem requerer, a um só tempo, atenção e cuidado, dando uma ideia da dimensão dessa assistência.

Daí a relevância que têm as representações construídas sobre si mesmo e sobre a doença. Dado o elemento cultural que todos carregam, conhecer essas representações que atravessam constantemente o sujeito significa abordar aspectos complexos que podem interferir de modo direto ou indireto no cuidado, no tratamento e na vida do paciente e seus familiares. ${ }^{10}$

Compreender como a doença oncológica mobiliza toda a dinâmica familiar faz com que a equipe de enfermagem, em especial a enfermeira, ao direcionar e planejar a assistência, possa fazê-lo de uma maneira mais abrangente, porém individualizada, de qualidade, e que contribua para um melhor enfrentamento da doença.

\section{A ENFERMAGEM NO PLANEJ AMENTO DA ASSISTÊNCIA AO PACIENTE COM CÂNCER}

A percepção, pela enfermeira, dos aspectos destacados anteriormente, pode oferecer subsídios para que planeje a assistência, de forma a buscar atender, dentro do possível, as demandas deste paciente. Contudo, é preciso compreender que estas necessidades são experiências subjetivas, que podem se expressar de modos diferentes, de acordo com os contextos e as culturas vividas por eles. A transição entre saúde e doença é uma experiência dinâmica e altamente individualizada.

$\mathrm{E}$, ao individualizar-se 0 atendimento e atentar-se para a forma singular de enfrentamento à doença, os determinantes sociais devem ser considerados porque os estilos de vida individual e familiar, na maioria das vezes, não correspondem a uma livre escolha dos indivíduos e famílias. ${ }^{11}$

Assim, é de fundamental importância a relação profissional-paciente no processo de adesão ao tratamento, visto que existem fatores aí envolvidos que são próprios do contexto onde o paciente está inserido. Por isso, as enfermeiras oncologistas precisam entender e fazer-se entender, e compartilhar com o paciente/família o momento do tratamento como sendo de troca e interação. 
Um dos aspectos que mais tem chamado à atenção quanto à avaliação dos serviços de saúde é o despreparo dos profissionais para lidar com a dimensão subjetiva que toda a prática de saúde supõe. Isso diz respeito também à dificuldade em lidar com a doença pelo próprio estigma que ela traz e pelo próprio itinerário terapêutico vivido pelo paciente e família. ${ }^{12}$

Nessa relação, a comunicação favorece a interação social e pode contribuir para uma maior compreensão e colaboração no tratamento, pois é um instrumento importante na construção da representação social, mediante o processo de interação que promove no grupo social., ${ }^{3,13}$

Ao ser responsável por uma equipe, a enfermeira deve assumir o compromisso de orientá-la e supervisioná-la, com vistas a assegurar a assistência requerida, utilizando conhecimentos fundamentados para respaldar suas ações e oferecer uma assistência individualizada, diferenciada e participativa.

Definir o tipo de cuidado a ser dispensado ao paciente requer da enfermeira a utilização do processo de enfermagem, objetivando implementá-lo de forma mais integrada, envolvendo também a família, de modo a planejar e executar a assistência alcançando as possíveis e reais necessidades do indivíduo. Assim, ao mantê-lo informado e consciente acerca das ações previstas para sua assistência poderá estabelecer o diálogo tão propício e necessário ao processo educativo.

Implica, ainda, identificar os aspectos que dizem respeito às limitações terapêuticas e organizacionais para a execução dessa assistência, considerando que as prioridades não devem recair apenas no manejo da doença, mas se estender ao ambiente construído ao seu redor. A responsabilidade da profissional se estende à tentativa de ouvir o paciente e enxergar além de sua palavra. ${ }^{10}$ Ou seja, precisa compreender como 0 câncer, e o próprio tratamento, podem provocar alterações nesse paciente e que trazem repercussões muito próprias, individuais, mas igualmente significativas e complexas.

Nessa linha de raciocínio, podemos dizer que o foco da intervenção em saúde, onde incluímos o planejamento da assistência de enfermagem, precisa ser deslocado do corpo em desequilibrio para os grupos sociais a partir das suas condições concretas - objetivas e subjetivas - de existência, avançando da valorização biológica para uma valorização da dimensão psicossocial. Contudo, isso deve ocorrer sem alienar o indivíduo do seu contexto de vida, pois, se essas dimensões forem tomadas como fatores isolados no sujeito, com o objetivo de ordenar a ação profissional, mantém-se uma situação de primazia do conhecimento técnico sobre o conhecimento do senso comum, assim como a relação de desigualdade ora existente entre eles. ${ }^{14}$

Em face disso, para garantir que as ações que viabilizam a atenção integral sejam executadas, é preciso uma visão clara da finalidade do trabalho de todos os profissionais envolvidos, visando 0 alcance dessa assistência, o que assume uma complexidade que vai além da qualidade dos profissionais que a executam. Isso porque a forma como se desenvolvem as relações sociais entre os indivíduos, decifrando, predizendo e antecipando as ações de determinado grupo no desenvolvimento de seu fazer cotidiano, são influenciadas pelas dimensões sociais aí imbricadas. ${ }^{15}$

Tal processo não se restringe apenas à relação enfermeira-paciente-família, mas aos demais profissionais que interagem com a mesma, com suas especificidades e demandas próprias, pois o trabalho em saúde requer o reconhecimento de que as atividades desenvolvidas pelos distintos profissionais se legitimam a partir da realização de cada trabalho especializado, ao tempo em que busca o equilíbrio entre as demandas desses profissionais e da população assistida. ${ }^{16}$

É preciso reconhecer que cada profissional é único, elabora um conhecimento prático, com significações próprias, ao mesmo tempo em que vive em grupo, partilha com ele um pensamento comum sobre sua prática, nesse caso, 0 planejamento da assistência, mas possui potencialidades e limitaç̃̃es geradas na complexidade dessa ação.

A atuação em oncologia requer da equipe de enfermagem mais que conhecimentos teóricos e práticos; exige o desenvolvimento de habilidades que possam nortear a sua atuação profissional, considerando as dimensões físicas, emocionais, sociais e espirituais dos pacientes sob sua responsabilidade, com uma doença crônica, com demandas contínuas e imprevisíveis.

Daí a importância de o profissional buscar o desenvolvimento de habilidades específicas exigidas para sua área de atuação. Especificamente em Oncologia, a busca por um curso de especialização é significativa, visto que na maior parte das instituições de ensino brasileiras, em nível de graduação em Enfermagem, o ensino da Cancerologia ainda é premente. $^{17}$

As práticas profissionais, vistas a partir das representações sociais, possibilitam um novo olhar, que não mais as considera estritamente técnica e cientificamente determinada, mas como expressão das subjetividades, que se constituem em um dado contexto social. Ou seja, implica conceber o duplo caráter assumido pelas práticas profissionais: o primeiro, como ação legitimada pelo conhecimento científico, e o segundo, como papel de construção da realidade, e, desse modo, como uma função da experiência pessoal, da ação criativa e do conhecimento do senso comum. ${ }^{12}$

\section{CONSIDERAÇÕES FINAIS}

A enfermeira e os demais profissionais de enfermagem, ao planejarem e programarem a assistência, podem contribuir com o paciente para ampliar sua capacidade para enfrentar o processo saúde-doença, diante do potencial que cada pessoa possui de se reorganizar e enfrentar situações de risco, através de um planejamento com base no levantamento de problemas e prescrição de cuidados fundamentados nas necessidades e possibilidades do outro, respeitando sua autonomia, suas 
crenças e valores, de modo a tornar a complexidade dos procedimentos o mais próximo possível de sua compreensão e participação no próprio cuidado.

Cumpre destacar que o tratamento do câncer pode ser prolongado, muitas vezes doloroso, limitante, e provocar mudanças significativas na vida pessoal, profissional e social de quem está doente e também de seus familiares e amigos, o que certamente requer uma rede de atenção que permita ao paciente dar seguimento ao seu tratamento. Estes aspectos estão destacados na Política Nacional de Atenção Oncológica, caracterizando a Atenção Integral à Saúde da Pessoa com Câncer, além de se configurarem como evidências em muitos estudos desenvolvidos por enfermeiras que atuam em oncologia no Brasil.

0 ensino da oncologia nos cursos de graduação em Enfermagem além de abordar os aspectos biomédicos, deveria incorporar os aspectos subjetivos do cuidado no processo de morrer, atenção às famílias, questões sobre autoimagem, aspectos éticos no final da vida, entre outros, e aqueles relacionados à atenção ao profissional que cuida desses pacientes. Parece não existir, por parte dessas instituições, uma preocupação com a formação dos alunos com enfoque nessa área, que ocorre ainda de forma incipiente.

Em outra perspectiva, dentro das organizações hospitalares, de um modo geral, não se observa uma preocupação marcante quanto aos aspectos emocionais dos profissionais de saúde, especialmente os de enfermagem, pelo tempo que dispendem na assistência, que ficam expostos frequentemente às situações de gravidade e perda de pacientes. Há necessidade de se discutir sobre a qualidade de vida e da morte de seus pacientes, mas também sobre as condições de trabalho (físicas e emocionais) que esses profissionais encontram para assisti-los.

Ao ponderarmos sobre as dimensões que estão imbricadas no planejamento da assistência ao paciente com câncer, temos ideia da amplitude da responsabilidade da enfermeira no seu papel profissional/sujeito, em um processo que envolve reconhecer a relevância da autonomia do ser cuidado/sujeito, buscando na assistência uma relação de integração, democrática, que permita ao outro expor suas habilidades, conhecimentos e deficiências, assim como ela própria as possui, mas que o respeite nas suas limitações, crenças e valores.

Estar ciente da importância do seu papel assistencial/ educativo permitirá que a enfermeira planeje a assistência de enfermagem mais condizente à realidade deste paciente/família, aceitando-os como uma unidade indissociável, que requer escuta, comprometimento, autonomia, liberdade, como cidadãos, independente da fase da vida em que se encontrem, pois se a doença e a saúde lhe conferem direitos, a proximidade da morte não os remove.

A subjetividade profissional implica rever necessidades e tecnologias de trabalho, além de respeitar o humano profissional e a clientela por ele atendida. 0 que se observa, muitas vezes, é que existe um despreparo do profissional de enfermagem para lidar com essa subjetividade. Isso requer uma atenção por parte das organizações e instituições de ensino, no sentido de prevenir o desgaste emocional desses profissionais e discentes, o que já foi comprovado em literatura, pelo nível de estresse e envolvimento com pacientes e familiares.

Diante do exposto, acreditamos que há espaço para expandir estas reflexões, de modo a possibilitar 0 reconhecimento das concretas condições de trabalho da enfermeira oncologista e os processos que exprimem e articulam a relação entre a objetividade da sua prática de planejamento e a subjetividade de cada profissional inserido neste grupo social, e de sua relação com esse tipo de paciente.

Esperamos que o conhecimento produzido com este estudo possa contribuir para suscitar novas discussões sobre o tema, visto que poucos trabalhos foram encontrados com abordagem semelhante, mas que se fazem necessários para identificar sua per tinência tanto na realidade dos serviços de saúde quanto na prática das enfermeiras.

\section{REFERÊNCIAS}

1. Ministério da Saúde (BR). Instituto Nacional de Câncer [homepage na Internet]. [citado 2007 nov 01]. Disponível em: http://www.inca.gov.br.

2. Fugita RMI, Farah OGD. O planejamento como instrumento básico do enfermeiro. In: Cianciarullo TI, organizador. Instrumentos básicos para o cuidar: um desafio para a qualidade da assistência. São Paulo (SP): Atheneu; 1996.

3. Moscovici S. A representação social da psicanálise. Rio de Janeiro (RJ): Zahar; 1978.

4. Atinkson SJ. Anthropology in research on the quality of health services. Cad Saude Publica, Rio de Janeiro, 1993 jul/set; 9(3): 283-99.

5. Ramos C, Carvalho JEC, Mangiacavalli MASC. Impacto e (i) mobilização: um estudo sobre campanhas de prevenção ao câncer. Cienc Saude Colet. 2007; 12(5): 1387- 396.

6. Maruyama SAT, CostaALC, Espírito Santo EAR, Bellato R, Pereira WR. 0 corpo e a cultura como lócus do câncer. Cogitare Enferm. 2006 maio/ ago; 11(2): 171-75.

7. Weihermann AMC. Usando um referencial cultural para cuidar de mulheres que tiveram câncer de mama. Texto\&Contexto Enferm. 2000; 9(2): 67382.

8. Oliveira AP, Gomes AMT. A estrutura representacional do câncer para seus portadores: desvelando seus sentidos e dimensões. Rev Enferm UERJ. Rio de Janeiro, 2008 out/dez; 16(4): 525-31. 
9. Ferraz AF, Oliveira CM, Matos SS, Costa TMPF, Cabral DL, Caldeira EM. 0 domicílio como cenário alternativo de apoio ao paciente oncológico. REME: Rev Min Enferm. 2006 out/dez; 10(4): 440-47.

10. Barbosa LNF, Francisco AL. A subjetividade do câncer na cultura: implicações na clínica contemporânea. Rev SBPH, Rio de Janeiro, 2007 jun; 10(1): 9-23.

11. Carvalho CSU. A necessária atenção à família do paciente oncológico. Rev Bras Cancerol 2008; 54(1): 87- 96.

12. Oliveira ACF. Incorporação da dimensão subjetiva do trabalho em saúde e dos princípios da humanização no processo de formação dos enfermeiros nos programas de residência e especialização oncológica do INCA. [dissertação] Rio de Janeiro (RJ): Escola Nacional de Saúde Sérgio Arouca, Fundação Oswaldo Cruz; 2007. 75p.

13. Jodelet $D$. Representações sociais: um domínio em expansão. Rio de Janeiro (RJ): EDUERJ; 2002. 236 p.

14. Oliveira DC. Representações sociais em saúde e educação. In: Oliveira DC, Campos PHF, organizadores. Representações sociais: uma teoria sem fronteiras. Rio de Janeiro (RJ): Museu da República, 2005. 170p.

15. Cruz EA. Práticas profissionais dos trabalhadores em central de material e esterilização: representações sociais da equipe de enfermagem. [tese]. Fortaleza (CE): Universidade Federal do Ceará; 2003. 214p.

16. Nunes IMN, Amâncio Filho, Moura MAV, Telles JL. 0 trabalho em saúde no contexto hospitalar: processos e necessidades como subsídios para a formação profissional. Esc Anna Nery. 2006 dez; 10(3): 509-13.

17. Rodrigues AB. Burn out e estilos de coping em enfermeiros que assistem pacientes oncológicos. [tese]. São Paulo (SP): Escola de Enfermagem, Universidade de São Paulo; 2006. 143 p. 